\title{
Compact setup for spin-, time-, and angle- resolved photoemission spectroscopy
}

Cite as: Rev. Sci. Instrum. 91, 063001 (2020); https://doi.org/10.1063/5.0004861

Submitted: 14 February 2020 . Accepted: 16 May 2020 . Published Online: 08 June 2020

(iD K. Bühlmann, (D) R. Gort, (D) A. Fognini, (D) s. Däster, (D) S. Holenstein, (D) N. Hartmann, (D) Y. Zemp, G. Salvatella, (D) T. U. Michlmayr, T. Bähler, (D) D. Kutnyakhov, K. Medjanik, G. Schönhense, (D) A. Vaterlaus, and (D) Y. Acremann

\section{ARTICLES YOU MAY BE INTERESTED IN}

Time- and momentum-resolved photoemission studies using time-of-flight momentum microscopy at a free-electron laser

Review of Scientific Instruments 91, 013109 (2020); https://doi.org/10.1063/1.5118777

High resolution time- and angle-resolved photoemission spectroscopy with 11 eV laser pulses

Review of Scientific Instruments 91, 043102 (2020); https://doi.org/10.1063/1.5139556

Time-resolved momentum microscopy with a $1 \mathrm{MHz}$ high-harmonic extreme ultraviolet beamline

Review of Scientific Instruments 91, 063905 (2020); https://doi.org/10.1063/5.0006531

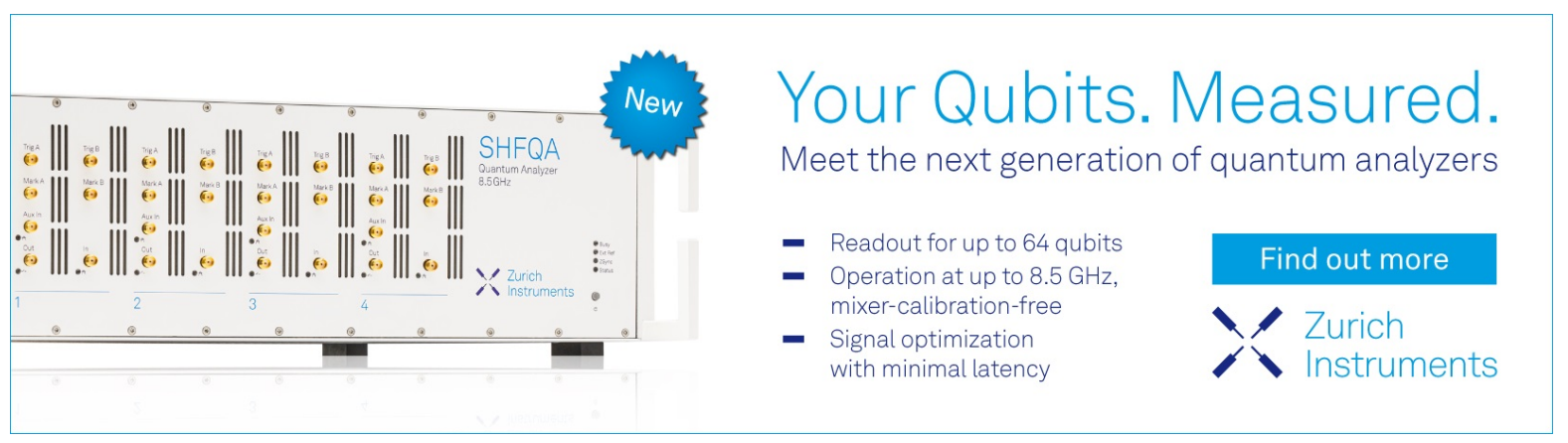




\title{
Compact setup for spin-, time-, and angle-resolved photoemission spectroscopy
}

\author{
Cite as: Rev. Sci. Instrum. 91, 063001 (2020); doi: 10.1063/5.0004861 \\ Submitted: 14 February 2020 • Accepted: 16 May 2020 • \\ Published Online: 8 June 2020
}

\begin{abstract}
K. Bühlmann, ${ }^{1, a)}$ (D) R. Gort, ${ }^{1,2}$ (D) A. Fognini, ${ }^{1,3}$ (D) S. Däster, ${ }^{1}$ (D) S. Holenstein, ${ }^{1,4}$ (D) N. Hartmann, ${ }^{1,5}$ (D) Y. Zemp, ${ }^{1}$ G. Salvatella, ${ }^{1}$ T. U. Michlmayr, ${ }^{1}$ (D) T. Bähler, ${ }^{1}$ D. Kutnyakhov, ${ }^{6,7}$ (D) K. Medjanik, ${ }^{6}$ G. Schönhense, ${ }^{6}$ A. Vaterlaus, and Y. Acremann ${ }^{1, b)}$ (D)
\end{abstract}

\author{
AFFILIATIONS \\ ${ }^{1}$ Laboratory for Solid State Physics, ETH Zurich, 8093 Zurich, Switzerland \\ ${ }^{2}$ European XFEL GmbH, Holzkoppel 4, 22869 Schenefeld, Germany \\ ${ }^{3}$ Single Quantum B.V., Molengraaffsingel 10, 2629 JD Delft, The Netherlands \\ ${ }^{4}$ Department of Physics, University of Zurich, Winterthurerstrasse 190, CH-8057 Zurich, Switzerland \\ ${ }^{5}$ Institute for Quamtum Electronics, ETH Zurich, 8093 Zurich, Switzerland \\ ${ }^{6}$ Institute of Physics, Johannes Gutenberg University of Mainz, 55128 Mainz, Germany \\ ${ }^{7}$ DESY Photon Science, Notkestrasse 85, 22607 Hamburg, Germany \\ a) Electronic mail: kevinbu@phys.ethz.ch \\ b) Author to whom correspondence should be addressed: acremann@solid.phys.ethz.ch
}

\begin{abstract}
We present a compact setup for spin-, time-, and angle-resolved photoemission spectroscopy. A $10 \mathrm{kHz}$ titanium sapphire laser system delivers pulses of $20 \mathrm{fs}$ duration, which drive a high harmonic generation-based source for ultraviolet photons at $21 \mathrm{eV}$ for photoemission. The same laser also excites the sample for pump-probe experiments. Emitted electrons pass through a hemispherical energy analyzer and a spin-filtering element. The latter is based on spin-polarized low-energy electron diffraction on an Au-passivated iridium crystal. The performance of the measurement system is discussed in terms of the resolution and efficiency of the spin filter, which are higher than those for Mott-based techniques.
\end{abstract}

\section{INTRODUCTION}

Optical techniques such as the different versions (longitudinal, transversal, polar, and complex) of the magneto-optical Kerr effect (MOKE) or magnetic second harmonic generation (mSHG) are most commonly used for probing the dynamic evolution of a solid's magnetization. Their widespread use is due to their relatively easy implementation. However, these methods probe the spin system in an indirect way. Therefore, their applicability can be limited, particularly if effects on the subpicosecond timescale are investigated. An example of this is the qualitative discrepancy between the results obtained by different optical techniques in spin-transport experiments.

The desire to gain insight into the spin-resolved band structure has driven the development of detection schemes that allow the recording of the emitted electron energy, momentum, and spin.
Formerly, most detection schemes were based on Mott scattering, where spin-orbit coupling results in a spin-dependent angular distribution after high energy scattering (typically $50 \mathrm{keV}$ ) on a gold foil. ${ }^{2,3}$ Mott polarimetry has two disadvantages when used for photoemission experiments. First, the process distorts the trajectories of the electrons such that the original angular and spectral distribution cannot be inferred. Therefore, it is usually necessary to preselect a certain window of energy and angle of the emitted electrons, which then results in a single measurement point. The current development of an imaging Mott spin detector ${ }^{4}$ may improve this issue. Second, Mott scattering suffers from a very low efficiency, as discussed in Sec. IV.

The advances in highly efficient low energy scattering methods with imaging (information-conserving) properties have facilitated spin filtering without loss of spectral and angular information. Therefore, these methods can be implemented in an 
angle-resolved photoemission spectroscopy (ARPES) setup without the need for any additional preselection element.

Application of the pump-probe scheme with ultrashort radiation pulses for electron emission and sample excitation completes the spin-, time-, and angle-resolved photoemission spectroscopy (STARPES) method.

\section{HIGH HARMONIC SOURCE}

High harmonic generation (HHG) is a very versatile source of ultrashort (down to attoseconds) radiation pulses from the ultraviolet (UV) up to soft $\mathrm{x}$ rays. ${ }^{6}$ Implementations are typically compact (tabletop) and, if a suitable driving laser system is already available, quite affordable. The generated pulses show high spatial and temporal coherence and are intrinsically synchronized to the laser system driving the HHG, which is a great advantage for measurements that require two or more pulses (e.g., pump-probe and streaking). For these reasons, HHG-based sources are widely used in ultrafast science.

The use of spin-resolved photoemission spectroscopy implies several requirements on the radiation characteristics:

- Spectral resolution can be achieved by the use of a single harmonic. A short driving wavelength $\lambda_{0}$ results in an increased line separation in the HHG spectrum. The use of $\lambda_{0}=400 \mathrm{~nm}$ leads to a separation of the lines of $\approx$ $6 \mathrm{eV}$. In this case, multilayer mirrors can be used to filter out a single line of the HHG spectrum, simplifying the setup.

- The desire to access the entire valence band leads to a lower photon energy limit $E_{\text {min }}$, which is roughly the sum of typical values of the work function $\Phi$ and valence band width $\Delta E_{v}$ plus a few $\mathrm{eV}$ to avoid coverage by the cascade of secondary electrons: $E_{\text {min }} \approx 5 \mathrm{eV}+10 \mathrm{eV}+5 \mathrm{eV}=20 \mathrm{eV}$.

- The spin-filtering process reduces the number of detectable electrons by roughly two orders of magnitude. Hence, we aim for a highly efficient conversion. This again points toward a rather short driving wavelength as the efficiency of HHG is proportional to $\lambda_{0}^{-\alpha}$, with $5<\alpha<6$.

A titanium sapphire amplified laser system (Legend Elite, Coherent) delivers pulses of $1 \mathrm{~mJ}$ energy and $20 \mathrm{fs}$ duration at a center wavelength of $800 \mathrm{~nm}$ with a repetition rate of $10 \mathrm{kHz}$. These properties in combination with the abovementioned demands led to a design that drives the HHG with the second harmonic of the original beam created in a $0.2 \mathrm{~mm}$ thick beta barium borate crystal. ${ }^{9,10}$ The seventh-order generated photons at an energy of $21 \mathrm{eV}$ are then selected for photoemission.

The $400 \mathrm{~nm}$ wavelength driving beam with a pulse energy of $\approx 100 \mu \mathrm{J}$ is focused by a lens of $20 \mathrm{~cm}$ focal length into the generation chamber, which is already part of the vacuum system. The generation occurs in an argon jet that intersects the beam perpendicularly at its focus. The jet is delivered by a $75 \mu \mathrm{m}$ wide nozzle, as shown in Fig. 1. The nozzle consists of a $5 \mathrm{~mm}$ long glass capillary. Variations in the backing pressure affect the source output via changes in the emission per unit volume, phase matching between the driving and generated beams, and absorption of UV

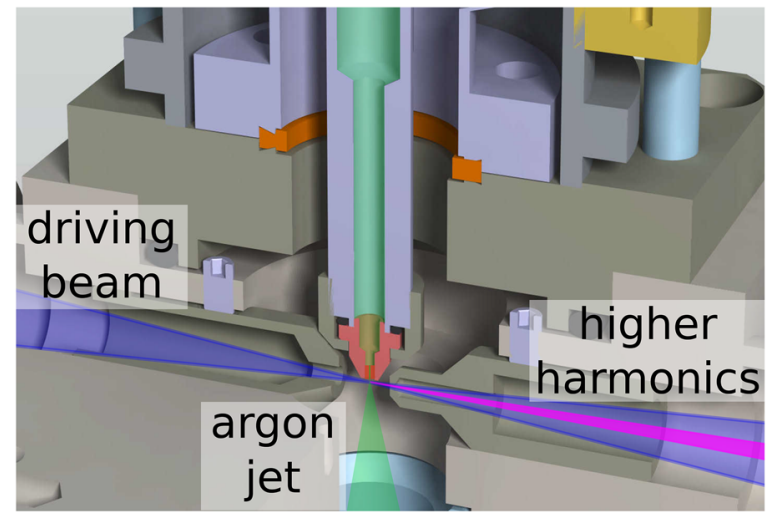

FIG. 1. Close-up CAD drawing of the high harmonic generation chamber. The driving beam (blue) comes from the left and intersects the argon jet (green), creating the higher harmonics (purple).

light by residual gas outside the interaction region. The optimal pressure in this setup is found to be $\sim 2$ bars at the input of the nozzle. Since argon is highly absorbing for UV radiation, its pressure after generation is reduced as quickly as possible. The jet, introducing a strong pressure gradient by itself, is directly pumped by using a pre-vacuum scroll pump. The beam path passes through two apertures that serve as differential pumping stages, whereas the volume in between them is pumped by using a separate turbo molecular pump.

The following small vacuum chamber hosts elements for filtering and refocusing of the divergent beam that still contains the driving wavelength and all the created higher harmonics. The operational pressure in this mirror chamber is typically $10^{-5} \mathrm{mbar}$. The first optic is a plane silicon wafer installed at a Brewster's angle of $80^{\circ}$ for the $400 \mathrm{~nm}$ light, absorbing most of it, whereas higher energetic radiation is reflected by $\approx 90 \%$. ${ }^{11}$ The use of the wafer is not compulsory, and the following elements are also capable of stopping the driving beam. However, experience has shown that a strong reduction in the beam power at this point is needed to protect the delicate parts further along the beam path. Next are two concave Bragg mirrors with focal lengths of $0.5 \mathrm{~m}$ and $1 \mathrm{~m}$ optimized for the reflection of $21 \mathrm{eV}$ radiation under an angle of $5^{\circ}$ to the surface normal. Each of these $\mathrm{Sc} / \mathrm{Si}$ multilayer mirrors is manufactured by the FraunhoferInstitut für Angewandte Optik in Jena for a reflectivity of $>30 \%$ at a photon energy of $21 \mathrm{eV}$. These mirrors first collimate and then refocus the beam onto the sample and provide spectral filtering. The final element before the UHV measurement chamber and the sample therein is a $75 \mathrm{~nm}$ thick, freestanding aluminum film that serves as vacuum barrier and a high-pass filter with a cutoff at $15 \mathrm{eV}$. The transmission of the Al filter is $\approx 80 \%$ according to Ref. 12 . Therefore, we estimate the total transmission for the UV beam from the source to be $6 \%$. However, the transmission may be reduced by the formation of an oxide film on the $\mathrm{Al}$ foil and contaminants on the optics.

We estimate an upper limit of the beam size on the sample by retracting the $\mathrm{Al}$ foil and imaging the $400 \mathrm{~nm}$ beam profile, leading to a focus diameter of $0.5 \mathrm{~mm}$. 


\section{DETECTION SETUP}

The sample is surrounded by a metal plate that acts as a potential plane and a pair of coreless coils connected to a high current pulse generator ${ }^{13}$ that sets the magnetization of the sample. This design leads to an environment with a homogeneous electric field and no magnetic field during measurements. A voltage can be applied to the sample and the potential plate to shift the whole photoelectron spectrum in terms of the kinetic energy.

The path of the electrons from the sample to the detector is schematically shown in Fig. 2. First, a hemispherical energy analyzer (HEA) (Phoibos 150, Specs) sorts the emitted electrons by energy and emission angle (and therefore momentum), creating a two-dimensional electron distribution with these two quantities as the axes.

Our spin filter relies on spin-polarized low-energy electron diffraction (SPLEED) on a crystal. ${ }^{14-16}$ In our setup, we use an Ir crystal(100) crystal under an angle of $\theta=45^{\circ}$. The geometry of the setup results in sensitivity along the sample's in-plane direction perpendicular to the incoming pump and probe beams. The chosen scattering energy is $\sim 6 \mathrm{eV}$, where we have an $\approx 0.5 \mathrm{eV}$ wide energy window with a high spin sensitivity. Since the pass energy of the HEA is higher than this (typically between $15 \mathrm{eV}$ and $25 \mathrm{eV}$ ), electrons need to be slowed down before they hit the iridium surface. The setup is shown in Fig. 3: an electrostatic lens system is used to image the output of the HEA onto the analyzer crystal. The following lens system images the reflected electrons onto the imaging electron detector.

The properties defined by a given set of applied voltages can be visualized if a regularly spaced thin slit aperture is placed in front of the HEA, leading to the transmission of electrons with emission angles corresponding to one of the openings. At the HEA output, this results in a distribution that consists of equally spaced, parallel lines. The distribution on the detector is shown in Fig. 4, confirming satisfactory imaging quality.

SPLEED is critically affected by the surface of the analyzer crystal, and even a fraction of a monolayer (ML) of adsorbates significantly reduces the efficiency. Therefore, the useful lifetime of a freshly prepared crystal surface is only several hours at a typical working pressure of $10^{-10}$ mbar. Fortunately, this issue can be

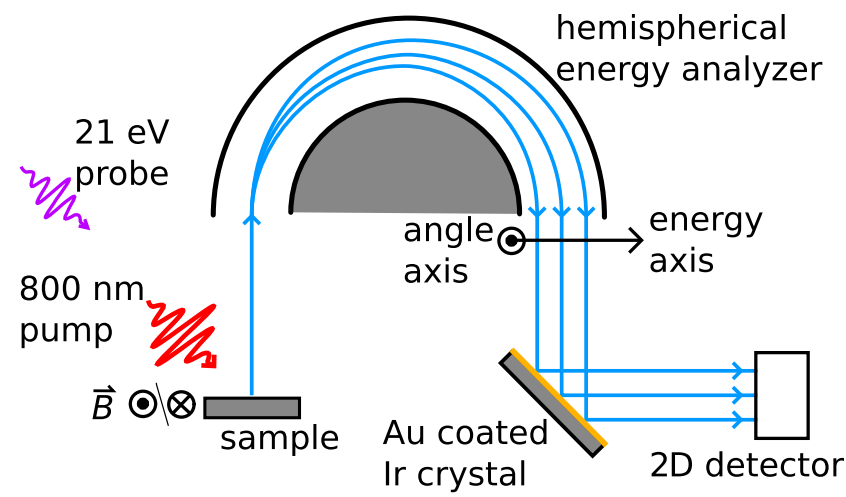

FIG. 2. Schematic overview of the detection setup.

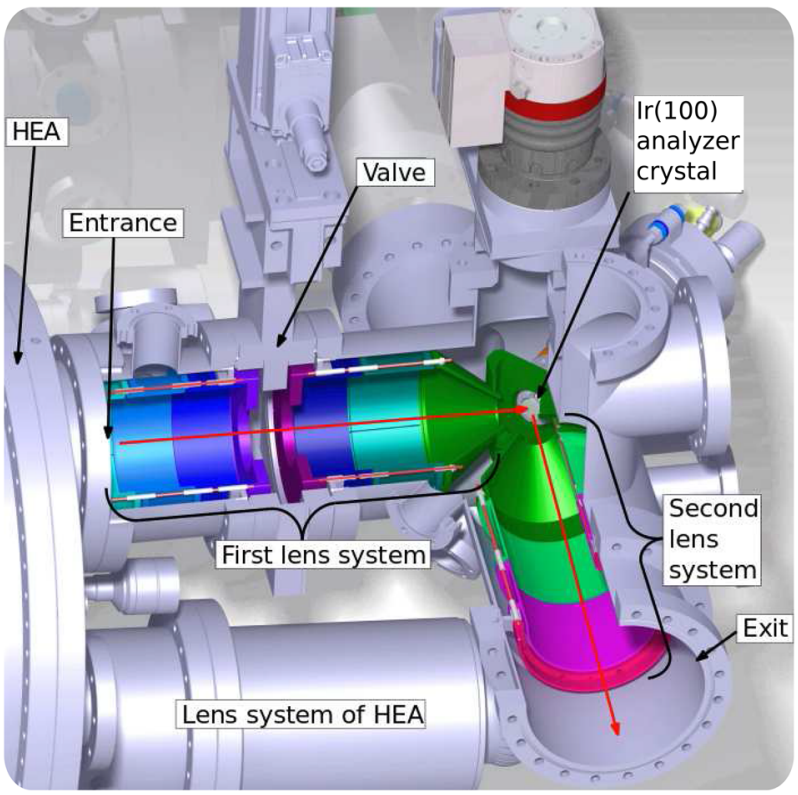

FIG. 3. Design of the electron lens system of the SPLEED detector. The electrons from the hemispherical energy analyzer (HEA, from Specs, type Phoibos 150) are imaged onto the Ir crystal. The reflected electron image is imaged onto the imaging electron detector.

resolved by protecting the iridium surface with one monolayer of gold. ${ }^{7,17,18}$ Exact coverage is achieved by deposition of an excessive amount of gold followed by a heating step that releases all but the last gold layer as it is bound stronger to iridium than to itself. We apply the procedure described in Ref. 17. A prepared crystal has a lifetime of at least several months (we never observed degradation but had to repeat the procedure after venting of the chamber).

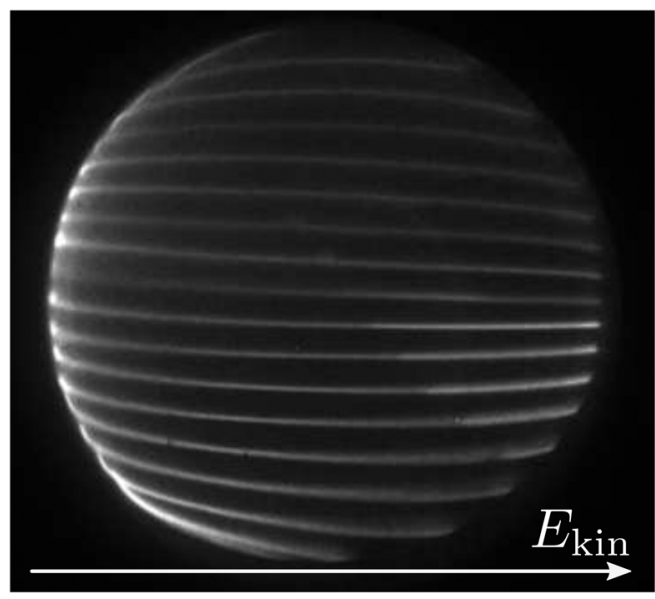

FIG. 4. Detected electron distribution for initially equally spaced, parallel lines along the energy dispersive direction at the HEA output. The line separation corresponds to an angle of $1^{\circ}$. 
For samples that can be magnetized, spin resolution is finally obtained by measuring twice with opposite magnetization directions. If this is not possible but spin information is still of interest (for example, for topological insulators), one can change the spinfiltering property of the crystal to introduce contrast by varying the scattering energy according to Ref. 7, controlled by the voltage applied to the crystal.

In order to obtain spin-resoled spectra from iron thin film samples, we apply a magnetic field pulse of 100 Oe field strength and 12 $\mu$ s length. By the pulse, the sample magnetization is saturated. Due to the use of field coils without the core, the magnetic field of the coil is zero during the measurement. After the pulse, electrons are detected for $10 \mathrm{~s}$ before the procedure is repeated with the opposite magnetic field direction. This sequence is repeated for a given integration time per data point. The read-out time of the camera is $\sim 500 \mathrm{~ms}$.

\section{PERFORMANCE}

The ratio between the measured asymmetry (in the count rates for opposite magnetization directions) and true spin polarization is called the Sherman factor (or function) $S$. The actual efficiency is better quantified by the figure of merit $F=S^{2} \cdot R$ as it is directly inversely proportional to the measurement time required for a given signal-to-noise ratio of the measured spin polarization. Here, $R$ is the reflectivity of the analyzer, i.e., the fraction of electrons incident on the crystal diffracted in the direction of the detector. In twodimensional detection schemes, $S, R$, and therefore $F$ are not just single numbers but functions that depend on the energy and scattering angle. In addition, one often includes the number of detectable distinguishable channels for a quantity that describes the performance of a detector. However, in our case, this is not applicable as the energy and angle resolution is mainly defined by the radiation used for the experiments. Furthermore, it is always necessary to heavily bin measured electron distributions to achieve sufficient statistics since the pump-probe delay adds a third dimension over which data is distributed. Hence, we consider the simultaneously detectable phase space as more significant than the number of channels.

We measure the spin polarization of low-energy secondary electrons from a thin iron film excited by an unpolarized electron beam at $2 \mathrm{keV}$. Comparison of the results with values found in the literature ${ }^{19,20}$ leads to a Sherman factor of roughly 0.5 over the whole simultaneously detected range. Measurements by Vasilyev et al. ${ }^{7}$ give us an estimate for $R$ on the order of one percent. This leads to a figure of merit of $\sim 2.5 \cdot 10^{-3}$, in line with the values reported in the literature ${ }^{17,18}$ and more than an order of magnitude higher than that for Mott spin polarimeters. ${ }^{21,22}$ The detected phase space depends on the exact electrostatic lens configuration but typically spans $0.5 \mathrm{eV}$ in energy and $0.4 \AA^{-1}$ in reciprocal space.

The setup can, in principle, resolve quite fine features in the emission angle down to a width of $0.1^{\circ}$. However, the spot size of the probe beam on the sample is limiting this capability. The effective resolution is typically an order of magnitude worse. For the given photon energy, this translates to $0.02 \AA^{-1}$.

The high harmonic source is bright enough to observe an energy shift in the photoelectrons' spectral distribution due to their mutual repulsion after emission. The occurrence of this space charge effect means that we are operating close to the optimal UV fluence. If we set the center of the energy window to $E_{f}-1 \mathrm{eV}$, we detect up to one electron per pulse, i.e., up to $10000 / \mathrm{s}$, as the system runs at $10 \mathrm{kHz}$.

The performance of the instrument can be demonstrated with experiments on a ferromagnetic iron film as well as a thin gold film. The iron sample under investigation is a $20 \mathrm{ML}$ single crystalline iron film deposited on a W(110) substrate by electron beam evaporation according to Ref. 23. Figure 5 shows the spin-resolved spectrum of the iron film. The number of detected majority electrons drops before the Fermi edge due to the shape of the density of states (DOS). In contrast, the minority bands extend beyond the Fermi energy. Therefore, the drop of the measured intensity at $E_{f}$ is dominated by the Fermi-Dirac function. In order to determine the energy resolution, we fit the measured spectrum of the minority electrons at the Fermi edge with

$$
I=I_{0} \frac{1}{\sqrt{2 \pi \sigma^{2}}} e^{\frac{-E^{2}}{2 \sigma^{2}}} * f(E, \mu, T) .
$$

Here, $I_{0}$ is a scaling factor to match the count rate, $E$ is the energy, $\mu$ is the chemical potential, $\sigma$ is the energy resolution, and $T=300 \mathrm{~K}$ is the temperature. $*$ denotes the convolution. From this fit (shown in Fig. 5), we obtain the energy resolution $\sigma=140 \mathrm{meV}$.

In Fig. 5, we also show the measured photoemission spectrum of a gold film deposited on $\mathrm{W}(110)$. The $5 \mathrm{~d}$ states are visible, which is a consequence of the photon energy of $21 \mathrm{eV}$.

For time-resolved experiments, the sample is excited by the $800 \mathrm{~nm}$ pump beam at a pump fluence of $5.6 \mathrm{~mJ} / \mathrm{cm}^{2}$. In Fig. 6, the resulting spectra of the Fe film above the Fermi edge are shown. The dashed curve shows the spectrum of the sample at a negative pumpprobe delay (where the pump pulse follows the probe pulse). The offset is due to the dark counts recorded by the detector. At positive delay times, the pump pulse causes excitation of nonthermal electrons, which is shown for the time when the pump and probe pulses perfectly overlap. The intensity has been scaled such that the

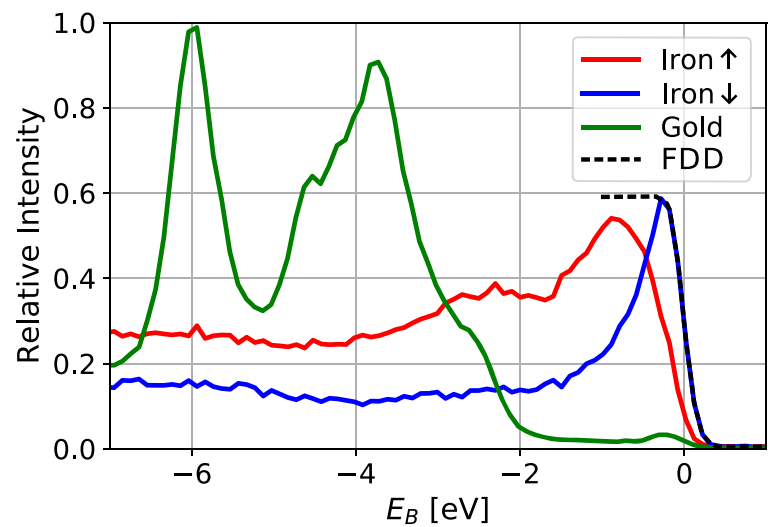

FIG. 5. Spin-resolved spectra of a Fe thin film on W(110). The minority spectrum (denoted Iron ${ }_{\downarrow}$ ) is cut at the Fermi edge by the Fermi-Dirac function. A fit of the edge by a Fermi-Dirac function convoluted with a Gauss function (labeled FDD) is used to determine the energy resolution of the setup. For comparison, the photoemission spectrum of an Au film on W(110) is shown, demonstrating the possibility of detecting electrons with a binding energy $>6 \mathrm{eV}$. 


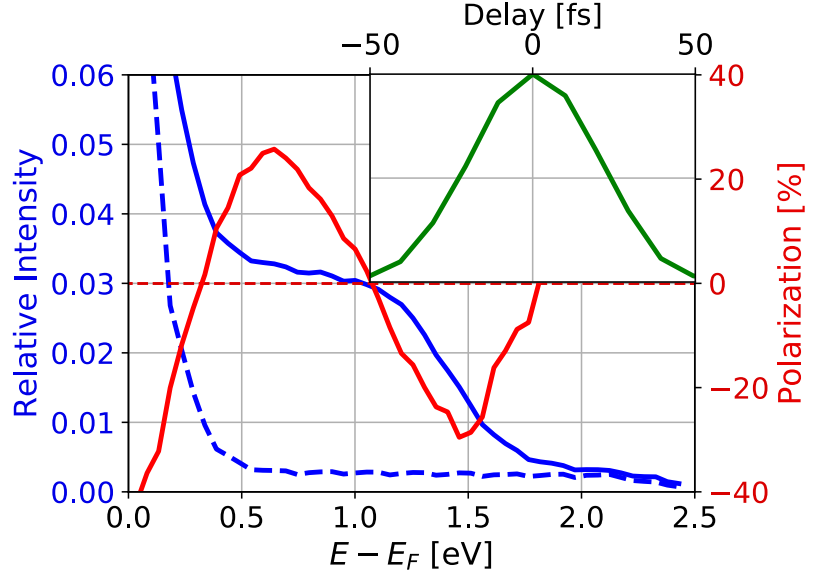

FIG. 6. Spectra of laser-excited Fe on W: the blue dashed curve shows the photoemission spectrum $100 \mathrm{fs}$ before the pump pulse. It follows approximately a Fermi-Dirac distribution (assuming the density of states is almost constant within the width of the Fermi edge) with an additional offset due to dark counts from the detector. The intensity has been scaled to be 1 at the maximum at $500 \mathrm{meV}$ below $E_{f}$. The blue solid curve shows the distribution of nonthermal electrons induced by the pump laser pulse at the exact temporal overlap between the pump and probe pulses. The spin polarization at the precise temporal overlap is shown in red. The inset shows the time dependence of the number of non-thermal electrons.

intensity maximum at $500 \mathrm{meV}$ below the Fermi energy is set to 1. The red curve shows the spin polarization of these non-thermal electrons. Even at $1.5 \mathrm{eV}$ above the Fermi edge, it is still possible to measure the spin polarization of nonthermal electrons. Since the photon energy of the pump beam is $1.5 \mathrm{eV}$, the negative polarization corresponds to the negative polarization at the Fermi edge. Details of these experiments can be found in Ref. 24.

The inset of Fig. 6 shows the time dependence of the number of non-thermal electrons measured 1.0-1.5 eV above the Fermi edge. From this measurement, we can estimate the temporal resolution by fitting a Gauss function to it. From the fit, we determine $\sigma=20 \mathrm{fs}$, corresponding to a full with at half maximum of $50 \mathrm{fs}$.

The same HEA, but equipped with a Mott spin detector, has been used for time- and spin-resolved experiments at the free electron laser FLASH in Hamburg. ${ }^{25}$ Notice that the integration time in both experiments was similar, and both experiments were performed at a probe fluence close to the space charge limit. The repetition rate of FLASH was 4000 pulses per second. However, it was only possible to measure the spin dynamics of the cascade electrons with a very limited data quality. In comparison, spin- and time-resolved spectroscopy of non-thermal electrons demonstrated here would not have been possible using the Mott spin polarimeter.

\section{CONCLUSIONS}

In this manuscript, we describe a spin-, time-, and energyresolved photoemission setup operating at a probing energy of $21 \mathrm{eV}$. The high figure-of-merit of the imaging SPLEED spin detector allows us to observe spin dynamics not only in an energy integrated mode but also in spectroscopy. It is possible to detect the spin polarization of non-thermal electrons generated by the $800 \mathrm{~nm}$ pump laser.
Momentum microscopes in combination with an imaging SPLEED spin filter will open the path to spin-, time-, and angleresolved band structure tomography at free electron lasers. ${ }^{2}$

\section{ACKNOWLEDGMENTS}

This work was supported by the Swiss National Science Foundation and ETH Zurich. We thank Gerald Rohde and Michael Bauer from the University of Kiel for advice on the design of the HHG source.

\section{DATA AVAILABILITY}

The data that support the findings of this study are available from the corresponding author upon reasonable request.

\section{REFERENCES}

${ }^{1}$ A. Alekhin, I. Razdolski, M. Berritta, D. Bürstel, V. Temnov, D. Diesing, U. Bovensiepen, G. Woltersdorf, P. M. Oppeneer, and A. Melnikov, "Magnetooptical properties of au upon the injection of hot spin-polarized electrons across Fe/Au(0 0 1) interfaces," J. Phys.: Condens. Matter 31, 124002 (2019).

${ }^{2}$ N. F. Mott, "The scattering of fast electrons by atomic nuclei," Proc. R. Soc. A 124, 425-442 (1929).

${ }^{3}$ T. J. Gay and F. B. Dunning, "Mott electron polarimetry," Rev. Sci. Instrum. 63, 1635-1651 (1992).

${ }^{4}$ V. N. Strocov, V. N. Petrov, and J. H. Dil, "Concept of a multichannel spinresolving electron analyzer based on mott scattering," J. Synchrotron Radiat. 22, 708-716 (2015).

${ }^{5}$ M. Kolbe, P. Lushchyk, B. Petereit, H. J. Elmers, G. Schönhense, A. Oelsner, C. Tusche, and J. Kirschner, "Highly efficient multichannel spin-polarization detection," Phys. Rev. Lett. 107, 207601 (2011).

${ }^{6}$ B. Dromey, M. Zepf, A. Gopal, K. Lancaster, M. S. Wei, K. Krushelnick, M. Tatarakis, N. Vakakis, S. Moustaizis, R. Kodama, M. Tampo, C. Stoeckl, R. Clarke, H. Habara, D. Neely, S. Karsch, and P. Norreys, "High harmonic generation in the relativistic limit," Nat. Phys. 2, 456-459 (2006).

${ }^{7}$ D. Vasilyev, C. Tusche, F. Giebels, H. Gollisch, R. Feder, and J. Kirschner, "Lowenergy electron reflection from Au-passivated $\operatorname{Ir}(001)$ for application in imaging spin-filters," J. Electron Spectrosc. Relat. Phenom. 199, 10-18 (2015).

${ }^{8}$ A. D. Shiner, C. Trallero-Herrero, N. Kajumba, H.-C. Bandulet, D. Comtois, F. Légaré, M. Giguère, J.-C. Kieffer, P. B. Corkum, D. M. Villeneuve, A. D. Shiner, C. Trallero-Herrero, N. Kajumba, H.-C. Bandulet, D. Comtois, F. Légaré, M. Giguère, J.-C. Kieffer, P. B. Corkum, and D. M. Villeneuve, "Wavelength scaling of high harmonic generation efficiency," Phys. Rev. Lett. 103, 073902 (2009).

${ }^{9}$ S. Eich, A. Stange, A. V. Carr, J. Urbancic, T. Popmintchev, M. Wiesenmayer, K. Jansen, A. Ruffing, S. Jakobs, T. Rohwer, S. Hellmann, C. Chen, P. Matyba, L. Kipp, K. Rossnagel, M. Bauer, M. M. Murnane, H. C. Kapteyn, S. Mathias, and M. Aeschlimann, "Time- and angle-resolved photoemission spectroscopy with optimized high-harmonic pulses using frequency-doubled Ti:Sapphire lasers," J. Electron Spectrosc. Relat. Phenom. 195, 231-236 (2014).

${ }^{10}$ M. Plötzing, R. Adam, C. Weier, L. Plucinski, S. Eich, S. Emmerich, M. Rollinger, M. Aeschlimann, S. Mathias, and C. M. Schneider, "Spin-resolved photoelectron spectroscopy using femtosecond extreme ultraviolet light pulses from high-order harmonic generation,” Rev. Sci. Instrum. 87, 043903 (2016).

${ }^{11}$ M. L. Scott, P. N. Arendt, B. J. Cameron, J. M. Saber, and B. E. Newnam, "Extreme ultraviolet reflectance degradation of aluminum and silicon from surface oxidation," Appl. Opt. 27, 1503-1507 (1988).

${ }^{12}$ See http://henke.lbl.gov/optical_constants for X-ray interactions with matter; accessed 06 April 2020.

${ }^{13}$ A. Fognini, T. U. Michlmayr, T. Bähler, C. Wetli, A. Vaterlaus, and Y. Acremann, "Magnetic pulser and sample holder for time- and spin-resolved photoemission spectroscopy on magnetic materials," Rev. Sci. Instrum. 83, 063906 (2012). 
${ }^{14}$ C. Tusche, M. Ellguth, A. A. Ünal, C.-T. Chiang, A. Winkelmann, A. Krasyuk, M. Hahn, G. Schönhense, and J. Kirschner, "Spin resolved photoelectron microscopy using a two-dimensional spin-polarizing electron mirror," Appl. Phys. Lett. 99, 032505 (2011).

${ }^{15}$ D. Kutnyakhov, P. Lushchyk, A. Fognini, D. Perriard, M. Kolbe, K. Medjanik, E. Fedchenko, S. A. Nepijko, H. J. Elmers, G. Salvatella, C. Stieger, R. Gort, T. Bähler, T. Michlmayer, Y. Acremann, A. Vaterlaus, F. Giebels, H. Gollisch, R. Feder, C. Tusche, A. Krasyuk, J. Kirschner, and G. Schönhense, "Imaging spin filter for electrons based on specular reflection from iridium (001)," Ultramicroscopy 130, 63-69 (2013), part of special issue: Eighth International Workshop on LEEM/PEEM.

${ }^{16}$ A. W. Fognini, "Ultrafast demagnetization: An electronic point of view," Ph.D. thesis, ETH Zurich, Switzerland, 2014.

${ }^{17}$ J. Kirschner, F. Giebels, H. Gollisch, and R. Feder, "Spin-polarized electron scattering from pseudomorphic Au on $\operatorname{Ir}(001)$," Phys. Rev. B 88, 125419 (2013).

${ }^{18} \mathrm{~S}$. Suga and C. Tusche, "Photoelectron spectroscopy in a wide $h v$ region from 6ev to 8kev with full momentum and spin resolution," J. Electron Spectrosc. Relat. Phenom. 200, 119-142 (2015), part of special issue: Special Anniversary Issue: Volume 200.

${ }^{19}$ J. Kirschner and K. Koike, "Spin polarization of secondary electrons from $\mathrm{Fe}(110)$ excited by unpolarized primary electrons," Surf. Sci. 273, 147-159 (1992).

${ }^{20}$ S. F. Mao, X. Sun, X. W. Fang, B. Da, and Z. J. Ding, "A Monte Carlo study of the spin polarization of secondary electrons," Surf. Interface Anal. 44, 703-708 (2012).

${ }^{21}$ T. Kohashi, M. Konoto, and K. Koike, "Systematic experiment of Mott scattering," Jpn. J. Appl. Phys., Part 1 45, 6468-6474 (2006).
${ }^{22}$ G. Ghiringhelli, K. Larsson, and N. B. Brookes, "High-efficiency spin-resolved and spin-integrated electron detection: Parallel mounting on a hemispherical analyzer," Rev. Sci. Instrum. 70, 4225-4230 (1999).

${ }^{23}$ S. Miesch, A. Fognini, Y. Acremann, A. Vaterlaus, and T. U. Michlmayr, "Fe on W(110), a stable magnetic reference system," J. Appl. Phys. 109, 013905 (2011).

${ }^{24}$ R. Gort, K. Bühlmann, S. Däster, G. Salvatella, N. Hartmann, Y. Zemp, S. Holenstein, C. Stieger, A. Fognini, T. Michlmayr, T. Bähler, A. Vaterlaus, and Y. Acremann, "Early stages of ultrafast spin dynamics in a 3d ferromagnet," Phys. Rev. Lett. 121, 087206 (2018).

${ }^{25}$ A. Fognini, T. U. Michlmayr, G. Salvatella, C. Wetli, U. Ramsperger, T. Bähler, F. Sorgenfrei, M. Beye, A. Eschenlohr, N. Pontius, C. Stamm, F. Hieke, M. Dell'Angela, S. de Jong, R. Kukreja, N. Gerasimova, V. Rybnikov, A. Al-Shemmary, H. Redlin, J. Raabe, A. Föhlisch, H. A. Dürr, W. Wurth, D. Pescia, A. Vaterlaus, and Y. Acremann, "Ultrafast reduction of the total magnetization in iron," Appl. Phys. Lett. 104, 032402 (2014).

${ }^{26}$ D. Kutnyakhov, R. P. Xian, M. Dendzik, M. Heber, F. Pressacco, S. Y. Agustsson, L. Wenthaus, H. Meyer, S. Gieschen, G. Mercurio, A. Benz, K. Bühlman, S. Däster, R. Gort, D. Curcio, K. Volckaert, M. Bianchi, C. Sanders, J. A. Miwa, S. Ulstrup, A. Oelsner, C. Tusche, Y.-J. Chen, D. Vasilyev, K. Medjanik, G. Brenner, S. Dziarzhytski, H. Redlin, B. Manschwetus, S. Dong, J. Hauer, L. Rettig, F. Diekmann, K. Rossnagel, J. Demsar, H.-J. Elmers, P. Hofmann, R. Ernstorfer, G. Schönhense, Y. Acremann, and W. Wurth, "Time- and momentum-resolved photoemission studies using time-of-flight momentum microscopy at a free-electron laser," Rev. Sci. Instrum. 91, 013109 (2020). 